\title{
Medienästhetik in Bildungskontexten
}

\author{
Zurück zur Übersicht über die Beiträge
}

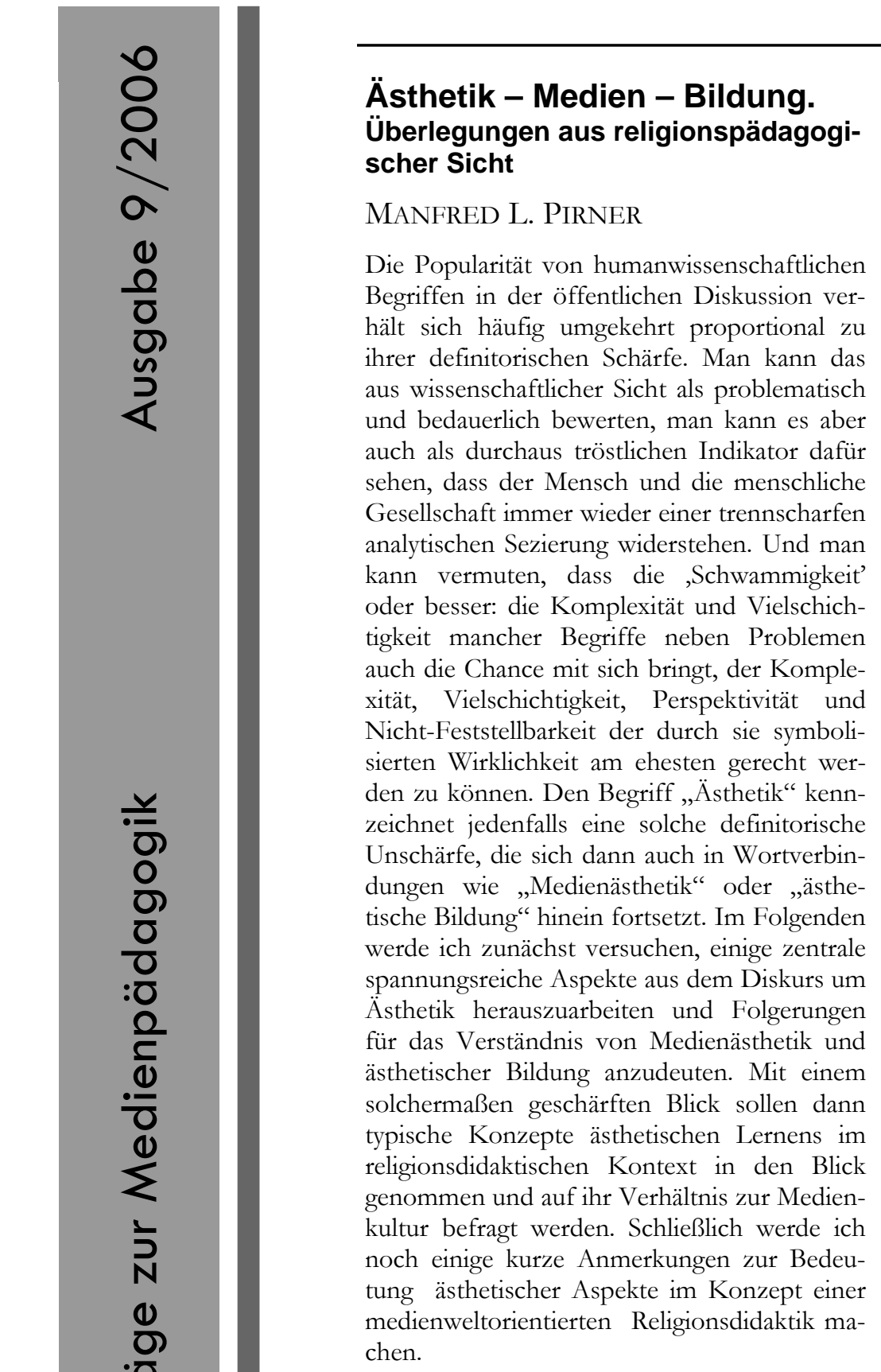

Ästhetik zwischen sinnlicher Wahrnehmung und Kunst

Der Ästhetik-Begriff ist in der Philosophiegeschichte vor allem mit der Theorie des Schönen, mit der Theorie der Kunst und mit der Theorie der sinnlichen Wahrnehmung in Verbindung gebracht worden. In (primär ,postmoderner") Philosophie und Teilen der Theologie ist, ebenso wie in Bereichen der Pädago- gik, seit den 1980er Jahren eine Tendenz wirksam geworden, Ästhetik wieder stärker - im Sinne ihres Begründers als wissenschaftliche Disziplin, Alexander Baumgarten („Aesthetica“ 1750/52) - auf die „aisthesis“ (Wahrnehmung) im umfassenden Sinn zu beziehen und sinnliche Erkenntnis als eigenständigen Erkenntnis- und Bewusstseinsmodus zu fassen, der sich damit auf die gesamte Wirklichkeit (nicht nur auf ,Schönes' oder ,Künstlerisches') beziehen kann. Die unterschiedliche Verhältnisbestimmung von ,ursprünglicher' Sinneswahrnehmung, ,künstlicher' sowie kreativkünstlerischer Versinnlichungen und deren Bewertungen (ästhetisches Urteil) spiegelt sich in heutigen Konzepten ästhetischen Lernens bzw. ästhetischer Bildung wider, wie unten gezeigt werden soll.

\section{Ästhetisierung zwischen Oberflä- che und Bedeutung}

Aus Trends der aktuellen gesellschaftlichen und wissenschaftlichen Diskussion lassen sich weitere spannungsreiche Facetten des Ästhetik-Begriffs erheben. Im Gefolge des „linguistic turn“" (sowie der schon fast wende-inflationär gebrauchten programmatischen Labels „cultural turn“, „iconic turn“, „pictural turn“ oder ,aesthetic turn“) haben wir gelernt, die Geringschätzung der Oberflächen $\mathrm{zu}$ überwinden und Bedeutungen nicht ,hinter', sondern in, mit und unter Sprache, Zeichen, Bildern und Inszenierungen zu finden. Gleichzeitig beobachten wir jedoch in unserer gegenwärtigen Gesellschaft eine meist als „Ästhetisierung der Lebenswelt" diagnostizierte Tendenz, Dinge eben nicht mehr nach ihrer Bedeutung, sondern vorrangig oder ausschließlich nach Kriterien des subjektiven Geschmacks zu beurteilen: „Findest du, dass die KreuzKette zu meinem Outfit passt ...", heißt eine der ,entscheidenden' Fragen, „... oder sollte ich heute lieber die Buddha-Ohrringe tragen?“. Bezogen auf die Medien: „Ich finde die religiösen Songs und Clips von Xavier Naidoo einfach geil; die bringen soviel feeling rüber!“ Oder: „Matrix I ist nach wie vor für mich der beste Film, weil der die besten special effects hat, zum Beispiel als der Neo wieder aufersteht." 
An diesen Beispielen wird deutlich, dass der Trend der Ästhetisierung sich als recht ambivalent darstellt: Einerseits kommen neue Ausdrucksmöglichkeiten und -qualitäten für ,Bedeutung' jenseits rationalistischer Engführungen in den Blick und es entstehen neue Bezüge oder Übergangsfelder zwischen kulturellen Bereichen wie Moral und Ästhetik oder Religion und Ästhetik - auf diese Aspekte hin könnte man auch die angeführten Beispiele noch einmal befragen, und es würde sich möglicherweise zeigen, dass doch ,noch mehr' ,hinter' den Aussagen steckt als bloße Geschmackspräferenzen (dann könnte man von einer „Tiefenästhetisierung“ im Sinne Wolfgang Welschs sprechen, vgl. Welsch 1993, 14). Andererseits scheint ein gewisser Trend zur Verweigerung von Bedeutung und zum SichZurückziehen ins ,Oberflächliche' unübersehbar. Hier werden Bedenken sowohl von der Pädagogik als auch von der (insbesondere protestantischen) Theologie und Religionspädagogik angemeldet werden, die sich stets gemeinsam gegen „Maulbrauchen“ ohne Verstehen und Vollzug äußerer Formen ohne innere Überzeugung sowie die damit verbundenen Gefahren der Manipulation gewandt haben. Pädagogisch und didaktisch hieße dies in der Konsequenz, sowohl die ästhetischen Gestalten ernster zu nehmen als auch auf der Nachfrage nach deren Bedeutungen und Funktionen zu bestehen.

\section{Wahrnehmung zwischen Objekt und Subjekt}

Im allgemeinen Gebrauch des Begriffs Ästhetik und ebenso in dem noch recht jungen Begriff der Medienästhetik zeigt sich als weitere Spannung die zwischen Objektgestalt (wie z. B. in der Rede von der Ästhetik eines Computerspiels oder eines Films) und subjektiver Rezeption. Fasst man Medienästhetik als „Theorie der Wahrnehmung unter den Bedingungen neuer technischer Medien“, so ist dabei - wie im Call zu diesem Themenheft angedeutet - die Subjekt-Perspektive betont, während die Frage der Objektgestalt sowie der aktiv-produktiven Gestaltung zumindest in die zweite Reihe rücken. Auch hier geht es wieder um eine fundamentale Fragestellung, deren Beantwortung sich bis hinein in konkrete pädagogische Konzepte auswirkt. Um es an einem Beispiel zu verdeutlichen: Wird das wahrnehmende Subjekt im Sinn einer konstruktivistischen Erkenntnistheorie als der entscheidende Faktor im Medienrezeptionsprozess veranschlagt, dann erscheinen (z. B. gewaltverherrlichende) Medieninhalte und -gestaltungen als wenig bedeutsam und problematisch - ein gewisser Trend, den ich in der qualitativen Medienrezeptionsforschung der letzten zwanzig Jahre wahrnehme und der me- medienpädagogische Konzepte beeinflusst hat. Wie teilweise auch in anderen Fachdidaktiken hat in der Religionsdidaktik - und darüber hinaus in der Praktischen Theologie - die auf Husserl und Merleau-Ponty zurück gehende phänomenologisch orientierte Erkenntnistheorie und Kunstphilosophie besondere Beachtung gefunden (vgl. v. a. Heimbrock 1998). Mit dem zentralen Konzept des „Erscheinens“ von Phänomenen liegt hier eine Theorie vor, die betont, dass Wahrnehmung und Erkenntnis sich gleichsam im ,Zwischenraum' zwischen Wahrnehmungsobjekt und wahrnehmendem Subjekt und deren jeweiligen Kontexten abspielen (vgl. für die Religionspädagogik Biehl 1998, für die Kunsttheorie Seel 2000; siehe auch für die Politik-Didaktik Besand 2004, 21). Beide Seiten, die Objektgestalt und ihre ,Rahmung' durch den Kontext sowie die subjektive Wahrnehmung mit all ihren Bedingungen und Voraussetzungen finden somit Beachtung und ermöglichen es, Wahrnehmung nicht nur als subjektive Konstruktionsleistung, sondern auch als Begegnung mit dem Anderen zu beschreiben und zu untersuchen (wobei sich hier auch Kompatibilitäten mit ,gemäßigten' bzw. differenzierten konstruktivistischen Ansätzen ergeben). Aus theologischer Perspektive gewinnt diese Sicht insofern eine besondere Relevanz, als sie ermöglicht, die für den christlichen Gottesglauben unaufgebbare „irreduzible Rezeptivität des Lebens“ (Schoberth 1998, 854) - auch als Korrektiv gegenüber dem neuzeitlichen Postulat des autonomen Subjekts - erkenntnistheoretisch zu denken.

In Bezug auf pädagogische Fragestellungen zur Medienästhetik erscheint mir eine solche Perspektive insofern besonders vielversprechend, weil sie zum einen die Lebenswelt der Kinder und Jugendlichen in umfassender Weise in den Blick kommen lässt und zum anderen ein Verständnis von Wahrnehmung nahe legt, das über die sinnliche ,Außenwahrnehmung' hinaus die innere Wahrnehmung mit ihren Assoziationen, Emotionen und Imaginationen - auch im Sinn einer „Wahrnehmung der Wahrnehmung“ - mit beachtet. Als eine pädagogische und didaktische Folgerung ergibt sich z. B., dass nicht nur die sinnliche Wahrnehmung und deren kognitivkonstruktive Verarbeitung, sondern auch der hilfreiche Umgang mit den eigenen Gefühlen und der eigenen Phantasie unterstützt sowie eine Kultivierung der „Wahrnehmung der Wahrnehmung“ angestrebt werden sollte - was Medienbildung letztlich auf Bildung im umfassenden und reflexiven Sinne hin orientiert.

\section{Soziale Konstruktion und intertex- tuelle Struktur von Wahrnehmung}

(Sozial-)konstruktivistische und semiotische Ansätze nehmen nach meiner Einschätzung in mancher Hinsicht noch deutlicher und differen- 
zierter als der phänomenologische Zugang die kulturelle Bedingtheit und Konstruiertheit von Wahrnehmung in den Blick und können diesen ergänzen, wenn auch m. E. nicht ersetzen. Insbesondere der aus der Literaturtheorie stammende und sowohl in der Semiotik als auch in den Cultural Studies aufgenommene Ansatz der Intertextualität enthält weiterführende Impulse. Nach ihren Hauptvertretern, Julia Kristeva und Michael Bachtin, umfasst er nicht nur die engere Frage von Beziehungen zwischen konkreten Texten (bzw. Zeichen), sondern in seiner weiteren Fassung auch die Beziehungen, die Wörter eines Textes mit den vorherigen Verwendungen dieses Wortes in anderen Texten und Situationen verbinden, so dass letztlich jeder Text als ein „Mosaik von Zitaten“ aufgefasst werden kann (Kristeva 1972, 245).

Der Fernsehwissenschaftler Lothar Mikos fasst die Bedeutung des Konzepts der Intertextualität für den Bereich der populären Medienkultur folgendermaßen zusammen: „Die Texte der Populärkultur stehen [...] in einem kulturellen Referenzsystem. Jeder Text der Populärkultur situiert sich so in einem bereits vorhandenen Universum der Texte, und die Position einzelner Texte verändert sich mit jedem neuen Text, der hinzukommt [...] Texte sind daher keine geschlossenen Einheiten, sondern offene, von zahllosen Referenzen, Zitaten und Einflüssen dynamisierte Prozesse. Ihre Bedeutung kann nur erschlossen werden, wenn sie auch auf andere Texte hin ,gelesen' bzw. gesehen' werden. Die subjektiven Rezeptionsgeschichten, also die Erfahrungen von Zuschauern mit Texten der Populärkultur als Teil ihrer Identität gehen in die Rezeption und Aneignung von Texten ein. Intertextualität ist also zugleich bestimmt durch alle Texte, die die Leser oder die Zuschauer an die Texte herantragen." (Mikos 1999, 6).

Der Vorzug des auf der Basis eines semiotisch erweiterten Textbegriffs verstandenen Konzepts der Intertextualität liegt in der gleichzeitigen Fokussierung des Objekts, des individuellen Aneignungsprozesses und ihrer jeweiligen kulturellen Bedingtheiten. Für die Religionsdidaktik eröffnet das Konzept insbesondere Chancen, Bezüge zwischen Medien und Religion differenziert in den Blick zu bekommen. Den Nachteil des intertextuellen Ansatzes wie der semiotischen Perspektive insgesamt sehe ich darin, dass hier ästhetische Wahrnehmung primär als Dechiffrierung von Codes verstanden und damit wie auch die Metapher des „Lesens von Texten“ andeutet - rationalistisch eng geführt zu werden droht. Demgegenüber wird im Folgenden die transrationale Seite von Ästhetik betont.

\section{Ästhetik als das Andere der Ratio}

Durch die Geschichte hindurch und vor allem seit der Romantik ist das Ästhetische stark mit dem Emotionalen und Irrationalen in Verbindung gebracht und teilweise programmatisch als
Gegenpol zu einem aufklärerisch-kognitivistischen und moralistischen Rationalismus verstanden worden. Da ästhetische Erfahrungen zugleich sehr viel subjektiver und individueller sind als etwa die Rezeption dogmatischer oder moralischer Grundsätze, die - dem Anspruch nach - auf der Basis von allgemeinmenschlichen Vernunftregeln formuliert sind, impliziert das Ästhetische tendenziell ein individualisierendes, anarchisches und autoritätskritisches Moment.

Bildungstheoretisch können sowohl in der Überwindung rationalistischer Engführung als auch in der Stärkung der Subjekte in deren Individualität besondere Chancen ästhetischer Bildung gesehen werden, die zugleich als Kriterien an die Beurteilung von medienästhetischen Gestaltungen und medienpädagogischen Bildungsprozessen herangetragen werden können: Ermöglichen Mediengestaltungen und das pädagogische Arbeiten mit und über Medien den Kindern und Jugendlichen ganzheitliche Wahrnehmungsfrei- und -spielräume oder werden sie auf die mechanisch-rationale Entschlüsselung ,eindeutiger' Codes festgelegt? Wird dem individuellen ästhetischen Urteil der Heranwachsenden ein Wert und Eigenrecht eingeräumt oder sollen sie zu einem bestimmten, z. B. - im Fall der Werbung - konsumdienlichen oder - im Fall des Schulunterrichts - künstlerisch-anspruchsvollen, Urteil ge- oder verführt werden?

Problematisch erscheint allerdings andererseits eine Emotionalisierung und Subjektivierung des Ästhetischen, das von kognitiver Bewusstmachung, rationaler Argumentation und kritischer Diskussion weit gehend abgekoppelt wird. In der Religionspädagogik hat schon vor einigen Jahren Dietrich Zilleßen skeptisch gefragt, ob die „Konjunktur ästhetischer Religionspädagogik darin begründet“ sei, „dass das interesselose Wohlgefallen als eine Möglichkeit angesehen wird, in die Mitte der Dinge, in ihr Zentrum als ihr Wesen vorstoßen" und so den „Verwicklungen des Wertens“ entgehen zu können (Zilleßen 1999, 75). Noch schärfer hat jüngst der religionspädagogische „Alt-68er“ Godwin Lämmermann die zunehmende ästhetische Affinität der Religionspädagogik kritisch als „ein höchst ambivalentes Phänomen“ bezeichnet, das zwischen Regression und Progression schwanke (Lämmermann 2005, 359): „Als regressiv hat sie dort zu gelten, wo sie zur Illusion einer unmittelbaren Wahrnehmungsmöglichkeit oder zum Revival der Romantik verkümmert ... Wir erleben die fröhliche Wiederauferstehung der Gefühlsreligiosität als Opposition zum denkenden Glauben mit seinen gesellschaftspolitischen Handlungsperspektiven unter dem Panier christlicher Freiheit und Subjektivität. ... Ästhetische und kritische Bildung scheinen in Gegensatz zu geraten." 
Ob diese Analyse im Hinblick auf Joachim Kunstmanns Ansatz (Kunstmann 2002; 2004), den Lämmermann hier vor allem im Visier hat, stimmt, sei dahin gestellt; sinnvoll und angebracht wird es jedenfalls sein, das Verhältnis zwischen Wahrnehmung und kritischer Reflexion, zwischen individuellem Geschmack und gesellschaftlicher Verantwortung, zwischen Ästhetik und Ethik neu auszuloten. Dies gilt insbesondere auch für den Bereich der Medien, wo neben und im Verbund mit ästhetischen Ansätzen kulturfunktionalistische Theorien über längere Zeit gesellschafts- und medienkritische Perspektiven in der Medienforschung und teilweise auch in der Medienpädagogik gedämpft haben (vgl. Niesyto/ Rath/Sowa 2006). Die Frage, ob überhaupt und inwieweit ein Konzept ästhetischer Bildung politisch oder unpolitisch ist, wird sich jedenfalls nicht nur für die Politik-Didaktik (vgl. Besand 2005), sondern ebenso für alle anderen Fachdidaktiken, auch und in besonderer Weise für die Religionsdidaktik stellen.

Angesichts der oben angedeuteten Ressentiments Lämmermanns gegenüber einer Ästhetik der Unmittelbarkeit und der ,subjektiven Evidenz" (360) lässt sich auf die spezifischen Chancen einer die elektronischen Medien einbeziehenden ästhetischen Bildung verweisen. Gerade die ästhetische Erfahrung einer medial immer perfekter simulierten und konstruierten Wirklichkeit (einschließlich deren Thematisierung in den und durch die Medien selbst) kann den heilsamen Zweifel an der Möglichkeit unmittelbarer Wirklichkeitserfahrung nähren, die Konstruiertheit aller Wahrnehmung im Sinn der oben bereits angesprochenen „Wahrnehmung der Wahrnehmung“ bewusst machen und somit einen als reflexiv und kritisch verstandenen Bildungsprozess unterstützen.

\section{Das rezeptive und das aktiv- konstruktive Moment von Ästhetik}

Die Fassung von Ästhetik als Theorie der Wahrnehmung steht in der Gefahr, die rezeptive Seite zu betonen und das aktiv-produktive ästhetische Handeln und Gestalten einschließlich seiner Wechselwirkungen mit dem Wahrnehmen zu vernachlässigen. Diese Gefahr zeigt sich in neueren Lexikon-Artikeln ebenso wie in theologischen und religionspädagogischen Überlegungen zur ,ästhetischen Erfahrung", in denen in aller Regel nur die rezeptive Seite dieser Erfahrung im Blick ist. Diese Gefahr scheint mir teilweise auch bei der neuerlichen Tendenz zu bestehen, Praktische Theologie und Religionspädagogik dezidiert als Wahrnehmungswissenschaften $\mathrm{zu}$ verstehen (vgl. Grözinger 1995, Biehl 1997), wenn und insofern die grundlagentheoretische Wahrnehmungsorientierung gegen die Handlungsorientierung ausgespielt wird.
Das weithin geschärfte und durch empirische Befunde unterstützte Bewusstsein für die starken aktiv-konstruktiven Anteile der Wahrnehmung kann sich in der didaktischen Konsequenz ambivalent auswirken: Es kann dazu beitragen, Aneignungsprozesse gerade durch aktivierende, handlungsorientierte Verfahren zu fördern; es kann aber auch dazu führen, sich mit der Initiierung einer rezeptiven Begegnung der Heranwachsenden mit bestimmten Unterrichtsgegenständen zu begnügen, unter dem Vorwand, jede Rezeption sei ja in sich bereits aktiv-konstruktiv. Grundsätzlich wird an den vielfältigen Wechselbeziehungen von Wahrnehmen und Handeln festzuhalten und insbesondere an die wahrnehmungsprägenden und -verändernden Effekte des (Alltags-)Handelns zu erinnern sein, wie sie vor allem Merleau-Ponty in seiner Phänomenologie der Wahrnehmung herausgearbeitet hat (Merleau-Ponty 1945/1974).

\section{Kleine Typologie religionsdidakti- scher Konzepte ästhetischen Lernens und ihr Verhältnis zur Medienkultur}

Die vorgetragenen Grundsatzüberlegungen können den Blick schärfen für die Profile, die Stärken und Schwächen sowie die Ferne und Nähe zur Medienkultur von typischen Ansätzen ästhetischer Bildung, die in der Religionsdidaktik auszumachen sind, die es aber vermutlich in ähnlicher Weise in anderen Fachdidaktiken gibt.

1. Ästhetisches Lernen durch ,unmittelbare" Sinneswabrnebmung

Hier wird in einer Art pädagogischer Gegenbewegung zur häufig mediatisierten oder instrumentell vermittelten Wahrnehmung unserer Medienkultur die „ursprüngliche“ „direkte“ Sinneserfahrung gesucht und geschult, z. B. in der Begegnung mit der Natur bzw. deren elementaren Bestandteilen wie Stein, Licht, Wasser, Luft, oder im Erleben des eigenen Körpers. So ist beispielsweise in dem interessanten, vor allem für die Grundschule entwickelten Konzept eines „bewegten Religionsunterrichts“ von Elisabeth Buck „das unmittelbare Erlebnis“ ein „wesentlicher Bestandteil“" (Buck 2001, 18), das durch körper- und sinnen-betonte didaktische Arrangements ermöglicht wird. Audiovisuelle Medien oder die Medienerfahrungen der SchülerInnen kommen in diesem Unterricht nicht vor.

Noch deutlicher erscheint die medienfeindliche Haltung in der sich als Wahrnehmungs- und Sprachschule verstehenden Symboldidaktik von Hubertus Halbfas, in der die SchülerInnen laut einem Schulbuchtext (!) dazu angeleitet werden sollen, ,dass wir uns gegen die Umklammerung durch die Medien wehren und uns ihren Gesetzen entziehen. Diese Gesetze beherrschen unser Denken und zerstören unsere Fähigkeit zu hoffen ..." (Halbfas 1991, 164). Der Umgang mit elementaren und religiösen Symbolen zielt bei Halbfas vor allem auf eine Spiritualität der medi- 
tativen Unmittelbarkeit und der inneren Erfahrung, die aus der Wahrnehmung der Doppeloder Mehrsinnigkeit der Dinge mittels des zu entwickelnden und $\mathrm{zu}$ fördernden „SymbolSinns" entstehen soll: Man sieht nur mit dem Herzen bzw. mit dem „dritten Auge“ gut. Auf diesem Weg zur Innerlichkeit scheint die bunte, grelle Medienwelt nur zu stören, obwohl sich gerade auch an ihrer symbolreichen Ästhetik das dritte Auge üben ließe.

\section{2. Ästhetisches Lernen durch qualitätsvolle Kunst}

Dieser religionspädagogische Typus versucht, traditionelle und moderne Kunstwerke zur Erschließung von existenziellen und religiösen Themen zu ,nutzen', wobei die „Qualitätsmerkmale anspruchsvoller Kunst" (Lange 2002, 249) als entscheidend angesehen werden, um Bildungsprozesse zu initiieren. Auch hier kann es einen gewissen oppositionellen Gestus gegen die als zu platt, künstlerisch minderwertig oder oberflächlich apostrophierte Ästhetik der populären Medienkultur geben, ja es werden im didaktischen Setting manchmal bewusst als künstlerisch wertvoll geltende Alternativen zu populären Medieninszenierungen angeboten. Und auch hier geht es u. a. darum, die Sinneswahrnehmung zu schärfen und zu erweitern, die durch Medienerfahrungen eingeengt, verzerrt oder abgestumpft ist: „Wir machen uns zu selten bewusst, wie unzureichend und manipuliert unsere Farbwahrnehmung ist, wie wenig geschärft unsere Sinne für Farbrelationen sind“ (Mertin/Wendt 2004, 76).

\section{3. Ästhetisches Lernen mit religiösen und kirchlichen Formen und Gestalten}

Die wahrgenommene Ästhetisierung der Lebenswelt und enorme Bedeutung von Medieninszenierungen führt bei diesem Typus dazu, die ,eigenen' ästhetischen Gestalten, also jene aus dem Bereich der Kirchen und der Religionen wieder ernster zu nehmen. Die Ästhetik der Medien wird hier vor allem unter diagnostischem Blickwinkel wahrgenommen: Sie zeigt an, was heutige Menschen anspricht oder was ihnen fehlt. Es geht nicht darum, sie zu kopieren oder sich auf sie zu beziehen, sondern vielmehr die ästhetischen Gestalten des Kirchenraums, der traditionellen religiösen Symbole sowie der kirchlichen Inszenierungen mit Verfahren ästhetischen Lernens wieder zu entdecken. So hat sich beispielsweise in den letzten Jahren der sehr erfolgreiche Ansatz einer „Kirchen(raum)pädagogik“" entwickelt, der die sinnlichen Erlebnisqualitäten kirchlicher Gebäude für religiöses Lernen fruchtbar machen will (vgl. Macht 2002, Rupp 2006). Der Bezug zur Medienkultur wird in diesem Ansatz bislang m. W. nicht hergestellt, obwohl es durchaus reizvoll und didaktisch sinnvoll erschiene, der Aufnahme der ästhetischen Gestaltqualitäten von Kirchen oder liturgischen Inszenierungen in der Werbung, in Videoclips oder in Kinofilmen nachzugehen.

\section{4. Ästhetisches Lernen als Grunddimension einer per- formativen Religionsdidaktik.}

Hatte die Diagnose der Ästhetisierung der Lebenswelt und der ästhetischen Dimension des Menschen im zuvor vorgestellten Ansatz zu einer neuen Wertschätzung der kirchlichen Formen und Orte geführt, so wird in diesem Typus die ästhetische Qualität des Unterrichts selbst neu betont. Der Unterricht selbst soll zum „Schauplatz Religion“ (so der Buchtitel von Klie/Leonhard 2003) werden, soll „Religion zeigen“ (ein weiterer Buchtitel, Dressler/MeyerBlanck 1998) bzw. zur theaterähnlichen Aufführung, zur „Performanz“ bringen, auch deshalb, weil man nicht über etwas reden oder reflektieren kann, was die meisten SchülerInnen gar nicht mehr ,aus eigener Anschauung' kennen. Die performative Religionsdidaktik stellt sich nicht als ein eigener Ansatz dar, sondern eher als Überbegriff für eine ganze Reihe von symboldidaktisch, semiotisch, phänomenologisch, gestalttheoretisch, konstruktivistisch oder poststrukturalistisch orientierten konzeptionellen Vorschlägen, denen allen die Einsicht gemeinsam ist, dass Religionsunterricht sinnlich und dramatisch inszeniert werden sollte. Die elektronischen Medien werden hierbei eher sporadisch und weniger grundsätzlich mit einbezogen. Der Schwerpunkt der kulturellen Bezüge liegt, wie bereits angedeutet, eher auf dem Theater und dem Spiel, ein zentrales Ziel ist das ästhetisch(schau-)spielerische Sich-Annähern an Spiritualität und Religion (wobei den SchülerInnen erfreulich viel ,Spielraum' für eigenes aktives und kreatives Handeln eingeräumt wird). Dies entspricht nun allerdings in gewisser Weise dem medienästhetischen Umgang mit Religion in der populären Kultur, wo Spiritualität und Glaube häufig im Modus der ästhetischen Inszenierung und des „Als-ob“ auftauchen: „Like a prayer“ (Madonna), „Like a believer“ (Marla Glen).

\section{5. Ästhetisches Lernen in und mit der Medienkultur}

Überblickt man die bisher vorgestellten vier Typen religionspädagogischer Konzepte ästhetischen Lernens, dann zeigt sich, dass durchweg die Chance gesehen wird, durch sie scientifischrationalistische Verengungen, wie sie durch Lebenswelt und schulische Bildung bedingt sind, aufzubrechen und damit auch neue $\mathrm{Zu}$ gänge zum Bereich des Religiösen zu fördern. Den (elektronischen) Medien und ihrer Ästhetik wird dabei allerdings kaum etwas Positives zugetraut; sie werden eher als Negativfolie für das pädagogisch Anzustrebende verwendet oder bleiben randständig. Damit besteht auch die bedenkliche Tendenz, der von Kindern und Jugendlichen bevorzugten Ästhetik die Beachtung und Berechtigung abzusprechen und ästhetisches Lernen entweder im Sinne natürlicher 
Sinnlichkeit oder im Sinne künstlerischer Qualität oder im Sinne traditionell-kirchlicher Symbolik gegen die populäre Medienästhetik anzustreben.

In den mittlerweile zahlreichen Versuchen, religionsdidaktisch an die populäre Medienkultur anzuknüpfen - die ich an anderen Stellen wiederholt zusammenfassend dargestellt habe (vgl. zuletzt in Pirner 2004a und 2004b) - wird wiederum die Einbeziehung von Elementen der Medienkultur in den Religionsunterricht bislang wenig im Sinne ästhetischen Lernens bzw. ästhetischer Bildung verstanden. Ansätze dazu gibt es vor allem bezogen auf die Werbung, wo immer wieder Motive aus dem Bereich der kirchlichen Kunst und Symbolik in häufig charakteristischen Transformationen auftauchen (vgl. Buschmann/Pirner 2003; Bickelhaupt 2005) sowie im Hinblick auf Kinofilme, deren religionspädagogische Erschließung Inge Kirsner und Michael Wermke explizit als „Schule des Sehens“ verstehen (Kirsner/Wermke 2005; 2004). Vor allem aber wird in Konzepten und Versuchen mit aktiver Medienarbeit die veränderte Wahrnehmung immer wieder als Lernerfolg konstatiert, so dass insbesondere auch hier von einer „Schule des Sehens“ (so z. B. Becker-Schmidt 2005) gesprochen werden kann.

Grundsätzlich haben m. E. die aufgeführten religionsdidaktischen Typen ästhetischen Lernens ihre je eigene Berechtigung, gerade auch in ihrer alternativen Stellung gegenüber der Medienkultur - solange sie diese nicht kategorisch aus dem Religionsunterricht ausschlieBen, sondern sich auch auf die Wahrnehmung der Medien beziehen lassen. Gerade durch die spannungsreichen Bezüge von ,natürlicher' Sinneswahrnehmung und ,künstlicher' Medienwahrnehmung, von ,innerer' und ,äußerer' Wahrnehmung, von Begegnungen mit ,ernsthafter' sowie religiöser Kunst und Erfahrungen mit unterhaltsam-populärer Medienkunst - so meine These - gewinnt die Wahrnehmung der SchülerInnen in einer Weise an Differenzierungsfähigkeit, Dynamik und Urteilsvermögen, dass Bildung im umfassenden Sinn von ästhetischer Bildung, MedienBildung und religiöser Bildung erreicht wird. Als profiliertestes Rahmenkonzept, innerhalb dessen diese Bezüge hergestellt werden konnten und können, hat sich in der Religionsdidaktik die Symboldidaktik, insbesondere in der Ausprägung von Peter Biehls „kritischer Symbolkunde“" erwiesen (vgl. Biehl 1999). Was meine eigenen Versuche zu einer „medienweltorientierten Religionsdidaktik“ angeht (Pirner 2001; 2006), müssen diese sicher im Hinblick auf die Dimension der Ästhetik und der ästhetischen Bildung weitergeführt und ergänzt werden. Hierzu will ich abschließend noch einige knappe Überlegun- gen anstellen.

\section{Medienästhetik und ästhetische Bildung im Rahmen einer medienweltorientier- ten Religionsdidaktik}

Kunstmann (2002) verweist zurecht darauf, dass sowohl Bildung als auch Religion eine ästhetische Basis haben. Bildung kann als „einen Sinn für etwas haben“, als „waches Aufgeschlossensein, als Staunenkönnen, Sehenkönnen mit den Augen des anderen“ (289) und - ich ergänze als Sich-selbst-von-außen-wahrnehmen-können beschrieben werden. Religion wiederum ist, nach der vielzitierten Auffassung von Friedrich Schleiermacher „Anschauen des Universums ... so alles Einzelne als einen Theil des Ganzen, alles Beschränkte als eine Darstellung des Unendlichen hinnehmen ..." (Über die Religion, zit. nach Gräb 2005, 378), sie ist eine bestimmte Art der Welt-Anschauung. Insofern ist ästhetische Bildung nicht lediglich ein Teilbereich von Bildung neben anderen, sondern eine grundlegende Dimension von Bildung generell und religiöser Bildung speziell.

Medienästhetik als Bereich wissenschaftlicher Forschung (vgl. z. B. Schnell 2000) macht uns darauf aufmerksam, wie die kulturelle Dominanz bestimmter Medien menschliche Wahrnehmung verändert hat und verändert, wie bestimmte ästhetische Eigentümlichkeiten und Standards im Bereich der populären Medien menschliche Wahrnehmung geprägt haben und prägen. Dadurch verändert sich auch das, was Bildung ausmacht und was als ästhetische Erfahrung oder als religiöse Erfahrung beschrieben werden kann. Und es ergeben sich, wie oben bereits angedeutet, neue Übergangsfelder zwischen Bildung, Ästhetik, Moral und Religion - Übergangsfelder, die neue Chancen und Zugänge eröffnen, aber angesichts von Vermengungen und Vermischungen auch neue Unterscheidungs- und Urteilsfähigkeiten erfordern. Von daher habe ich vorgeschlagen, vier Aufgabenund Bezugsfelder einer medienweltorientierten Religionsdidaktik zu unterscheiden und auf einander zu beziehen, von denen die Medienästhetik eines darstellt (vgl. Pirner 2006, 336):

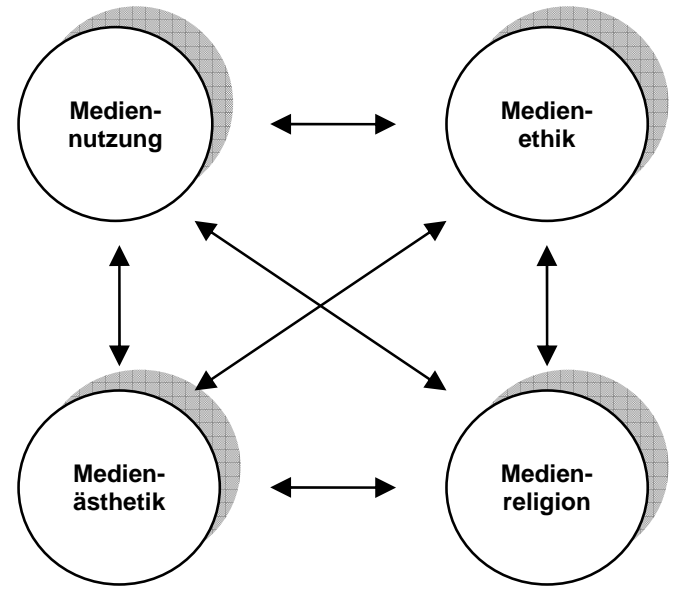


Mit Wilhelm Gräb sehe ich die zentrale Aufgabe der heutigen Religionspädagogik und -didaktik darin, die Kunst zu vermitteln, „die es braucht, um die ,gelebte Religion' der Individuen kritisch über sich selbst zu verständigen und in ihren lebensführungspraktischen, ethischen Konsequenzen konstruktiv $\mathrm{zu}$ formulieren“ (Gräb 2005, 377). Dazu gehört aber zunächst einmal, die Wahrnehmung zu erweitern und $\mathrm{zu}$ dynamisieren, den ,Sinn für das Religiöse' zu fördern, nämlich für die religiösen Dimensionen und Affinitäten eigener Wahrnehmungen, Einstellungen und Überlegungen ebenso sensibel zu werden wie für die vielfältigen Gestalten des Religiösen in der Kultur. In den populären Medien findet sich dieser ,Sinn für das Religiöse' immer wieder und zugleich werden die entsprechenden ästhetischen Inszenierungen hier auch unterschiedlichen Funktionalisierungen und Ideologisierungen unterworfen. In beiderlei Hin-Sicht, bezüglich der phänomenologisch-hermeneutischen Erschließung als auch bezüglich der kritischen Auseinandersetzung kann medienweltorientierter Religionsunterricht zur Wahrnehmungsbildung beitragen, so dass - wie ich das ab und zu selbst erlebt habe - hoffentlich noch viele SchülerInnen am Ende einer Unterrichtsstunde sagen: „So hab” ich das noch nie gesehen!“

\section{Literatur}

Becker-Schmidt, Anne: Das Videodrama als Schule des Sehens, in: Kirsner, Inge / Wermke, Michael (Hg.): Religion im Kino. Religionspädagogisches Arbeiten mit Filmen, Jena: IKS Garamond 2005, 221-239.

Besand, Anja: Angst vor der Oberfläche. Zum Verhältnis ästhetischen und politischen Lernens im Zeitalter Neuer Medien, Schalbach/Ts.: Wochenschau Vlg. 2004.

Bickelhaupt, Thomas: Kunst für's Volk. Kunstgeschichtliche Zitate in der Werbung der Printmedien, München: kopaed 2005.

Biehl, Peter: Festsymbole. Zum Beispiel: Ostern. Kreative Wahrnehmung als Ort der Symboldidaktik, Neukirchen-Vluyn: Neukirchener Vlg. 1999.

Biehl, Peter: Der phänomenologische Ansatz in der deutschen Religionspädagogik, in: Heimbrock, Hans-Günter (Hg.): Religionspädagogik und Phänomenologie. Von der empirischen Wendung zur Lebenswelt, Weinheim: Dt. Studien-Verl. 1998, 15-46.

Biehl, Peter: Wahrnehmung und ästhetische Erfahrung. Zur Bedeutung ästhetischen Denkens für eine Religionspädagogik als Wahrnehmungslehre, in: Grözinger, Albrecht / Lott, Jürgen (Hg.): Gelebte Religion, Rheinbach 1997.

Buck, Elisabeth: Bewegter Religionsunterricht, Göttingen: Vandenhoeck \& Ruprecht 3. Aufl. 2001.
Buschmann, Gerd / Pirner, Manfred L.: Werbung, Religion, Bildung, Frankfurt a. M. 2003.

Dressler, Bernhard / Meyer-Blanck, Michael (Hg.): Religion zeigen. Religionspädagogik und Semiotik, Münster: Lit 1998.

Gräb, Wilhelm: Ästhetische und religiöse Erfahrung in der Religionspädagogik, in: Zeitschrift für Pädagogik und Theologie 57 (2005), 375385.

Grözinger, Albrecht: Praktische Theologie als Kunst der Wahrnehmung, Gütersloh: Gütersloher Verl.-Haus 1995.

Halbfas, Hubertus: Religionsbuch für das neunte und zehnte Schuljahr, Teil 1, 9. Schuljahr, Düsseldorf 1991.

Heimbrock, Hans-Günter (Hg.): Religionspädagogik und Phänomenologie. Von der empirischen Wendung zur Lebenswelt, Weinheim: Dt. Studien-Verl. 1998.

Kirsner, Inge / Wermke, Michael (Hg.): Religion im Kino. Religionspädagogisches Arbeiten mit Filmen, Jena: IKS Garamond 2005.

Kirsner, Inge / Wermke, Michael (Hg.): Gewalt. Filmanalysen für den Religionsunterricht, Göttingen: Vandenhoeck \& Ruprecht 2004.

Klie, Thomas / Leonhard, Silke (Hg.): Schauplatz Religion. Grundzüge einer performativen Religionspädagogik, Leipzig: Evang. Verl.-Anst. 2003.

Kristeva, Julia: Probleme der Textstrukturation, Köln 1972.

Kunstmann, Joachim: Religion und Bildung. Zur ästhetischen Signatur religiöser Bildungsprozesse, Gütersloh: Kaiser / Gütersloher Verl.-Haus 2002.

Kunstmann, Joachim: Religionspädagogik, Tübingen / Basel: Francke 2004.

Lämmermann, Godwin: Religionspädagogik zwischen politischer und ästhetischer Signatur. Eine nicht ganz unpolemische Auseinandersetzung zur Rettung der Ästhetik vor den Ästheten, in: Zeitschrift für Pädagogik und Theologie 57(2005), H. 4, 358-368.

Macht, Siegfried: Kirchenräume begreifen. 70 Bausteine für Kirchenbesuch und Klassenzimmer, Lahr: Kaufmann 2002.

Merleau-Ponty, Maurice: Phänomenologie der Wahrnehmung (1945), Frankfurt a. M.: Suhrkamp 1974.

Mertin, Andreas / Wendt, Karin: Mit zeitgenössischer Kunst unterrichten. Religion - Ethik Philosophie, Göttingen: Vandenhoeck \& Ruprecht 2004 .

Mikos, Lothar: Erinnerung, Populärkultur und Lebensentwurf. Identität in der multimedialen Gesellschaft, in: Medien praktisch 23. Jg., 1999, H. 1, 4-8. 
Niesyto, Horst / Rath, Matthias / Sowa, Hubert (Hg.): Medienkritik heute. Grundlagen, Beispiele und Praxisfelder. Reihe Medienpädagogik interdisziplinär Band 5, Verlag kopaed, München 2006. (im Erscheinen)

Pirner, Manfred L.: Besondere Chancen einer medienweltorientierten Religionsdidaktik, in: M. Wermke / G. Adam / M. Rothgangel (Hg.), Religion in der Sekundarstufe II. Ein Kompendium, Göttingen: Vandenhoeck \& Ruprecht 2006, 328-356.

Pirner, Manfred L.: Religiöse Mediensozialisation. Empirische Studien zu Zusammenhängen zwischen Mediennutzung und Religiosität bei SchülerInnen und deren Wahrnehmung durch LehrerInnen, München: kopaed 2004 (a).

Pirner, Manfred L.: Einführung, in: Ders./ Breuer, Thomas (Hg.), Medien - Bildung Religion, München 2004, 11-18 (2004 b).

Pirner, Manfred L.: Fernsehmythen und religiöse Bildung, Frankfurt a. M.: GEP 2001.

Rupp, Hartmut (Hg.): Handbuch der Kirchenpädagogik. Kirchenräume wahrnehmen, deuten und erschließen, Stuttgart: Calwer 2006.

Schnell, Ralf: Medienästhetik. Zu Geschichte und Theorie audiovisueller Wahrnehmungsformen, Stuttgart u. a.: J.B. Metzlersche Verlagsbuchhandlung 2000.

Schoberth, Wolfgang: Art. Ästhetik, II. Theologisch, in: Religion in Geschichte und Gegenwart (RGG), Bd. 1 (1998), 853-854.

Seel, Martin: Ästhetik des Erscheinens, Frankfurt a.M.: Suhrkamp 2003.

Welsch, Wolfgang (Hg.): Die Aktualität des Ästhetischen, München: Fink 1993.

Zilleßen, Dietrich: Phänomenologie oder semiotische Religionspädagogik. Eine Alternative?, in: Klie, Thomas (Hg.): Spiegelflächen. Phänomenologie - Religionspädagogik - Werbung, Münster 1999, 72-89.

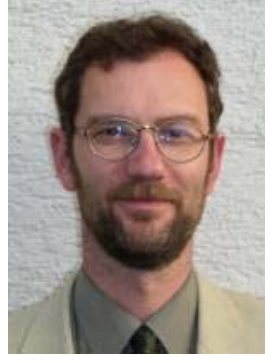

Prof. Dr. MANFred L. PIRNeR

Professor für Evangelische Theologie / Religionspädagogik an der Pädagogischen Hochschule Ludwigsburg

Arbeitsschwerpunkte:

Theologische Hermeneutik und Theorie der Kultur und der Medien; Musik und Religion, Sprachphilosophie und Theologie; Medienpädagogik und Religionspädagogik; Medienerziehung im Religionsunterricht 